\title{
Identificación de estresores en las prácticas clínicas y evolución de los mismos en una promoción de alummos de enfermería
}

\author{
López González JM. ${ }^{1}$, Orbañanos Peiro L. ${ }^{2}$, Cibanal Juan ML. ${ }^{3}$ \\ Sanid. mil. 2013; 69 (1): 13-21; ISSN: 1887-8571
}

\begin{abstract}
RESUMEN
Objetivo: Este estudio tiene como objetivo el identificar las fuentes de estrés y observar la evolución de la intensidad con que afectan a los alumnos de enfermería a lo largo de los tres años de carrera. Material y método: Estudio observacional, longitudinal y de carácter prospectivo de una cohorte de alumnos de enfermería a los que se investigó para observar y medir los factores causantes de estrés (estresores) que se generaron durante sus prácticas clínicas, y la evolución de los mismos a lo largo de sus tres años de carrera. Se utilizó el cuestionario KEZKAK, cuestionario bilingüe de estresores de los estudiantes de enfermería en las prácticas clínicas. Se trata de un cuestionario validado para medir específicamente los estresores que aparecen en los estudiantes de enfermería durante su formación práctica. Resultados y discusión: La incidencia de mayor a menor preocupación de los alumnos en sus prácticas clínicas en relación con los 9 factores o subescalas como fuentes generadoras de estrés fue: F1-falta de competencia, F4-impotencia e incertidumbre, F2-contacto con el sufrimiento, F5-no controlar la relación con el enfermo, F7-dañarse en la relación con el enfermo, F9-sobrecarga, F3-relación con tutores y compañeros. Así mismo, la evolución de la intensidad en la afectación, en términos generales, aumentó desde primero a tercero debido a la distribución de los rotatorios de prácticas ya que, los servicios especiales se concentraron durante el último curso académico. Conclusión: Las principales fuentes de estrés que afectan a los alumnos de nuestra escuela coinciden en cuanto a intensidad y prioridad con los estudios precedentes usados como referencia. Sin embargo, en general, difieren de los mismos, en cuanto a que van aumentando en intensidad al transcurrir cada curso como consecuencia de la distribución de los rotatorios de prácticas a lo largo de los tres años de carrera, ya que los rotatorios por los servicios que podían generar más tensiones en los alumnos (oncología, urgencias, coronarias, UCI, psiquiatría) se realizan en el último año; cosa que no ocurre en los estudios con los que se comparan nuestros resultados.
\end{abstract}

PALABRAS CLAVE: Estudiantes de enfermería, prácticas clínicas, estrés.

Identification of stressors in clinical practice and their evolution in a promotion of nursing students

SUMMARY: Aims: This study aims were to identify sources of stress and observe the evolution of the intensity affecting nursing students over the three-year degree. Material and Methods: Observational and longitudinal study of prospective nature of a nursing students cohort who are investigated to observe and measure the stress (stressors) that were generated during their clinical practice, and their evolution over his three year degree. KEZKAK questionnaire (bilingual questionnaire stressors of nursing students in clinical practice) was used. This is a validated questionnaire to measure the stressors that appear specifically in nursing students during their practical training. Results and Discussion: The incidence of high to low concern of students in their clinical practices relating to the 9 factors or subscales as generators of stress was: F1-lack of competition, F4-helplessness and uncertainty, F2-contact with suffering, F5-not control the relationship with the patient, F7-harmed by the relationship with the patient, F9-overload, F3-relationships with tutors and peers. Likewise, the intensity changes in the assignment, in general, increase from first to third. Conclusion: In light of the results and their discussion, we can conclude by saying that the main sources of stress affecting students in our school match in intensity and priority with previous studies used as reference. However, in general, differ from them, in that they are increasing in intensity at each elapse following the course of the rotary distribution practices throughout the stroke three years, as services by rotary that could create more tensions in students (oncology, emergency coronary ICU, psychiatry) are made in the last year, something that does not occur in studies that compare our results.

KEY WORDS: Nursing students, Clinical practice, Stress.

${ }^{1}$ Cap. Enfermero. Unidad Médica Aérea de Apoyo al Despliegue (UMAAD). Base

Aérea de Torrejón. Madrid. España.

2 Cte. Enfermero. Inspección General de Sanidad de la Defensa. Unidad de Enfermería. Madrid. España.

${ }^{3}$ Catedrático. Universidad de Alicante. Facultad de Enfermería. España.

Dirección para correspondencia: Juan Manuel López González. Unidad Médica Aérea de Apoyo al Despliegue (UMAAD). Base Aérea de Torrejón. 28850 Torrejón de Ardoz. Madrid.jlopgo2@oc.mde.es

Recibido: 23 de octubre de 2012

Aceptado: 20 de noviembre de 2012

\section{INTRODUCCIÓN}

El estrés académico afecta a variables tan diversas como el estado emocional, la salud física o las relaciones interpersonales, pudiendo ser vivenciadas de forma distinta por diferentes personas. A partir de la revisión de estudios sobre el tema, se pueden distinguir tres tipos de efectos: en el plano conductual, en el plano cognitivo y en el plano fisiológico ${ }^{1}$. 
En el plano conductual, el estilo de vida de los estudiantes se ve modificado según sufren las presiones propias del ámbito académico, adoptando hábitos insalubres ${ }^{2}$ : exceso en el consumo de cafeína, tabaco, sustancias psicoactivas como excitantes e incluso, en algunos casos, tranquilizantes ${ }^{3}$.

En el plano cognitivo, los patrones emocionales y de valoración de la realidad varían sustancialmente desde un periodo previo a, por ejemplo, la realización de los exámenes hasta el momento posterior al conocimiento de las calificaciones ${ }^{4}$. Respecto al plano psicofisiológico hay estudios que ponen de manifiesto la incidencia del estrés académico en el sistema inmunitario, demostrado por una depresión del mismo y, por tanto, por una mayor vulnerabilidad del organismo ante las enfermedades ${ }^{5}$.

Los alumnos de Enfermería viven con mucha ilusión y expectativas el comienzo de las prácticas clínicas. Pero también es cierto que las viven con cierto temor ${ }^{6}$. Tras las expectativas y temores se encuentra como motor, el deseo ${ }^{7}$. En un estudio realizado con médicos de asistencia primaria, Tizón ${ }^{8}$ observó que los deseos subyacentes a la labor de estos eran: el deseo de ayudar, deseo de aceptar y el deseo de ser aceptado. Estos deseos generan muchas de las expectativas y temores relacionados con la actividad profesional y, en la mayoría de los casos, se convierten en fuentes de estrés. Se podría decir que las expectativas de ayuda que alimenta el estudiante generan, junto con la inexperiencia, la preocupación de no responder o responder inadecuadamente a lo que se espera de un profesional de enfermería.

Zupiría y cols. concluyen, tras la aplicación del cuestionario Kezkak de estresores en una población de 86 alumnos de enfermería, que las principales fuentes de estrés, tanto al principio como al final de la carrera son, en orden de importancia: falta de competencia, impotencia e incertidumbre, dañarse en la relación con el enfermo, implicación emocional, no controlar la relación con el enfermo, contacto con el sufrimiento y relación con los compañeros 9 .

El gran volumen de trabajo que el curriculo presenta, a veces tiene implicaciones severas en el bienestar y calidad de vida de los estudiantes. Estos ingresan en la escuela, un mundo que le es desconocido, y se enfrentan a ritmos y responsabilidades intensas, que pueden mermar su fuerza y resistencia, ya que la carrera de enfermería tiene un fuerte componente de enseñanza clínica, una pesada carga horaria y una fuerte concentración de evaluaciones escritas ${ }^{10}$.

\section{JUSTIFICACIÓN}

Centrándonos en las prácticas clínicas, tenemos que convenir que el entorno sanitario suele proporcionar muchos estímulos generadores de estrés: el contacto con el paciente, los distintos medios donde se va a producir, la dinámica de trabajo, la falta de seguridad, la inmersión en un mundo nuevo con reglas y particularidades propias desconocidas en gran medida, los miedos y tabúes en relación con la enfermedad, la invalidez y la muerte, la necesidad de establecer relaciones con diversos profesionales sanitarios, así como el hecho de tener que desempeñar un nuevo rol para el que aún no están completamente preparados ${ }^{11}$.

Canalizar adecuadamente los sentimientos que generan estas tensiones puede ser la piedra angular para el normal desarrollo y crecimiento formativo, así como para evitar que sus mecanismos de afrontamiento se vean tan afectados que no cumplan adecuadamente con su función en la gestión personal de las dificultades.

Sería necesario conocer pues, las situaciones percibidas como más estresantes por los estudiantes en el desarrollo de sus prácticas clínicas, para poder intervenir con actividades de entrenamiento en estrategias de afrontamiento específicas, para la reducción de las tensiones generadas por los estresores más comunes. De este modo, creo que podríamos conseguir que reaccionen de una forma adaptativa a esos estímulos estresantes para evitar que puedan afectar negativamente a su formación práctica y a su propia salud.

Una vez identificados los estresores se estudió su evolución a lo largo de los tres años de carrera, y se estableció una comparación con dos estudios de similares características ${ }^{12,13}$.

\section{OBJETIVOS}

\section{Objetivo general}

Facilitar la adquisición de pautas de afrontamiento al estrés por medio de la identificación de los estresores más importantes y su momento de aparición durante las prácticas clínicas.

\section{Objetivos específicos}

- Identificar los principales estresores que influyen en la aparición de tensiones durante las prácticas clínicas en los alumnos de enfermería de la Universidad San Pablo CEU.

- Observar la evolución de lo estresores identificados en una misma promoción de alumnos, y la aparición de otros nuevos a lo largo de la carrera, según estos van adquiriendo experiencia ante la adquisición de nuevas responsabilidades.

\section{MATERIAL Y MÉTODO}

Estudio observacional prospectivo longitudinal de una cohorte de alumnos de enfermería a los que se investigó para observar y medir los factores causantes de estrés (estresores) que se generaron durante sus prácticas clínicas, y la evolución de los mismos a lo largo de sus tres años de carrera.

Para esto se utilizó el cuestionario KEZKAK, cuestionario bilingüe de estresores de los estudiantes de enfermería en las prácticas clínicas ${ }^{14}$. Se trata de un cuestionario validado para medir específicamente los estresores que aparecen en los estudiantes de enfermería durante su formación práctica. Este instrumento tiene una consistencia interna alta $(\alpha$ de Cronbach $=0,95)$ y una alta fiabilidad. El cuestionario es de tipo Likert y consta de 41 ítems en los que se pregunta al alumno por la preocupación que le genera cada una de las situaciones planteadas, repitiéndolo en cada uno de los tres cursos (tablas 1 y 2). Se puede optar entre cuatro respuestas (0: no preocupa nada; 1 : preocupa algo; 2 : preocupa bastante; 3: preocupa mucho). Posteriormente se pueden obtener: una puntuación total y una agrupación de los estresores en nueve subescalas o factores que indican distintas fuentes de estrés: F1Falta de competencia, F2 - Contacto con el sufrimiento, F3 - Relación con tutores y compañeros, F4 - Impotencia e incertidumbre, F5 - No controlar la relación con el enfermo, F6 - Implicación 
Tabla 1. Ordenación de los 41 ítems (estresores) del cuestionario KEZKAK según promedio total, de mayor a menor, con eliminación (trama de distinto color) de los ítems con promedio total menor de 1,50.

\begin{tabular}{|c|c|c|c|c|c|c|c|c|}
\hline Estresor & Nada & Algo & Bastante & Mucho & $\begin{array}{l}\text { Total } \\
(120)\end{array}$ & $\begin{array}{l}\text { Prom 1. } \\
(\mathrm{n} 1=48)\end{array}$ & $\begin{array}{l}\text { Prom 2. } \\
(\mathrm{n} 2=36)\end{array}$ & $\begin{array}{l}\text { Prom 3. } \\
(n 3=36)\end{array}$ \\
\hline 2 «Hacer mal mi trabajo y perjudicar al paciente» & 0 & 1 & 2 & 3 & 2,46 & 2,40 & 2,50 & 2,47 \\
\hline 16 «Confundirme de medicación» & 0 & 1 & 2 & 3 & 2,34 & 2,19 & 2,33 & 2,50 \\
\hline 15 «Pincharme con una aguja infectada» & 0 & 1 & 2 & 3 & 2,19 & 2,23 & 2,17 & 2,17 \\
\hline 4 «Hacer daño psicológico al paciente» & 0 & 1 & 2 & 3 & 2,17 & 2,04 & 2,28 & 2,19 \\
\hline 36 «Recibir órdenes contradictorias» & 0 & 1 & 2 & 3 & 2,15 & 1,83 & 2,31 & 2,31 \\
\hline $17 \ll$ Meter la pata» & 0 & 1 & 2 & 3 & 2,13 & 2,04 & 2,06 & 2,31 \\
\hline 6 «Hacer daño físico al paciente» & 0 & 1 & 2 & 3 & 2,12 & 2,06 & 2,25 & 2,06 \\
\hline 3 «Sentir que no puedo ayudar al paciente» & 0 & 1 & 2 & 3 & 2,09 & 1,90 & 2,08 & 2,28 \\
\hline 26 «Recibir la denuncia de un paciente» & 0 & 1 & 2 & 3 & 2,06 & 1,63 & 2,33 & 2,22 \\
\hline 20 «Encontrarme en alguna situación sin saber qué hacer» & 0 & 1 & 2 & 3 & 2,01 & 1,90 & 2,08 & 2,06 \\
\hline 29 «Encontrarme ante una situación de urgencia» & 0 & 1 & 2 & 3 & 1,93 & 1,94 & 1,97 & 1,89 \\
\hline 38 «No encontrar al médico cuando la situación lo requiere» & 0 & 1 & 2 & 3 & 1,91 & 1,77 & 1,81 & 2,14 \\
\hline 9 «Tener que dar malas noticias» & 0 & 1 & 2 & 3 & 1,88 & 1,75 & 1,86 & 2,03 \\
\hline 40 «Que el paciente toque ciertas partes de mi cuerpo» & 0 & 1 & 2 & 3 & 1,85 & 1,67 & 1,83 & 2,06 \\
\hline 13 «Contagiarme a través del paciente» & 0 & 1 & 2 & 3 & 1,84 & 1,88 & 1,81 & 1,83 \\
\hline 5 «No saber cómo responder a las expectativas de los pacientes» & 0 & 1 & 2 & 3 & 1,83 & 1,58 & 2,00 & 1,92 \\
\hline 27 «Tener que estar con la familia del paciente cuando éste se está muriendo» & 0 & 1 & 2 & 3 & 1,73 & 1,56 & 1,92 & 1,72 \\
\hline 35 «La sobrecarga de trabajo» & 0 & 1 & 2 & 3 & 1,70 & 1,46 & 1,75 & 1,89 \\
\hline 1 «No sentirme integrado/a en el equipo de trabajo" & 0 & 1 & 2 & 3 & 1,66 & 1,25 & 1,72 & 2,00 \\
\hline 7 «No saber cómo responder al paciente» & 0 & 1 & 2 & 3 & 1,64 & 1,38 & 1,67 & 1,89 \\
\hline 14 «Que un paciente que estaba mejorando comience a empeorar» & 0 & 1 & 2 & 3 & 1,62 & 1,52 & 1,67 & 1,67 \\
\hline 23 «No poder llegar a todos los pacientes» & 0 & 1 & 2 & 3 & 1,62 & 1,29 & 2,03 & 1,53 \\
\hline 18 «Ver morir a un paciente» & 0 & 1 & 2 & 3 & 1,54 & 1,42 & 1,64 & 1,56 \\
\hline 34 «Tener que trabajar con pacientes agresivos» & 0 & 1 & 2 & 3 & 1,46 & 1,40 & 1,44 & 1,53 \\
\hline 8 «Que me afecten las emociones del paciente» & 0 & 1 & 2 & 3 & 1,44 & 1,27 & 1,47 & 1,58 \\
\hline 31 «Tener que estar con un paciente al que se le ha ocultado una mala noticia» & 0 & 1 & 2 & 3 & 1,44 & 1,21 & 1,50 & 1,61 \\
\hline 32 «Tener que realizar procedimientos que duelen al paciente» & 0 & 1 & 2 & 3 & 1,36 & 1,31 & 1,28 & 1,50 \\
\hline 10 «Tener que hablar con el paciente de su sufrimiento» & 0 & 1 & 2 & 3 & 1,35 & 1,25 & 1,39 & 1,42 \\
\hline 22 «Que mi responsabilidad en el cuidado del paciente sea importante» & 0 & 1 & 2 & 3 & 1,34 & 1,06 & 1,47 & 1,50 \\
\hline 21 «mplicarme demasiado con el paciente» & 0 & 1 & 2 & 3 & 1,26 & 1,10 & 1,22 & 1,44 \\
\hline 24 «Que el paciente no me respete» & 0 & 1 & 2 & 3 & 1,25 & 1,00 & 1,33 & 1,42 \\
\hline 11 «Que el paciente me trate mal» & 0 & 1 & 2 & 3 & 1,24 & 0,69 & 1,39 & 1,64 \\
\hline 30 «Tener que estar con un paciente con el que es difícil comunicarse» & 0 & 1 & 2 & 3 & 1,19 & 1,00 & 1,17 & 1,39 \\
\hline 12 «La relación con los profesionales de la salud» & 0 & 1 & 2 & 3 & 1,18 & 1,06 & 1,14 & 1,33 \\
\hline 25 «La relación con la venia» & 0 & 1 & 2 & 3 & 1,18 & 1,02 & 1,28 & 1,25 \\
\hline 33 «No saber cómo «cortar» al paciente» & 0 & 1 & 2 & 3 & 1,13 & 1,08 & 1,11 & 1,19 \\
\hline 37 «Que un enfermo del otro sexo se me insinúe» & 0 & 1 & 2 & 3 & 1,12 & 0,67 & 1,22 & 1,47 \\
\hline 19 «La relación con el profesor responsable de prácticas de la escuela» & 0 & 1 & 2 & 3 & 0,90 & 0,77 & 0,83 & 1,08 \\
\hline 39 «Tener que estar con un paciente terminal» & 0 & 1 & 2 & 3 & 0,88 & 0,85 & 0,89 & 0,89 \\
\hline 28 «La relación con los compañeros estudiantes de enfermería» & 0 & 1 & 2 & 3 & 0,76 & 0,73 & 0,64 & 0,92 \\
\hline 41 «Las diferencias entre lo que aprendemos en clase y que vemos en las prácticas & 0 & 1 & 2 & 3 & 1,15 & 1,00 & 1,22 & 1,22 \\
\hline
\end{tabular}

emocional, F7 - Dañarse en la relación con el enfermo, F8 - En enfermo busca una relación íntima, F9 - Sobrecarga.

La puntuación obtenida en cada factor o subescala tiene rangos diferentes debido a que cada factor está constituido por un número diferente de ítems (tabla 3). Como queremos distinguir cuales son los factores más estresantes, se ha procurado obtener una puntuación ponderada dividiendo la puntuación obtenida por el número de ítems de cada factor. De esta manera el resultado obtenido se encuentra dentro de un rango entre 0 y 3 , al igual que las opciones de respuesta de cada ítem.

En todos los casos, tanto al observar cada uno de los 41 ítems por separado o agrupados en la 9 subescalas, se consideró que la situación era generadora de estrés cuando la puntuación obtenida en el ítem correspondiente era de 1,5 o superior.

En cuanto a la comparación entre cursos, los valores de la encuesta fueron tratados como variables cuantitativas. Como sus distribuciones vulneraban el supuesto de normalidad se empleó como índice de tendencia central la mediana (Md) y de dispersión la amplitud intercuartílica (IQR). El efecto se valoró mediante la diferencia de medianas. Para comparar los resultados cuantitativos de cada item en función de los 3 cursos se ha usado un test no paramétrico, el test de Kruskal Wallis (KW), que «equivale a un ANOVA para distribuciones no normales». Cuando la p de ese test resultó $<0,05$ entonces se buscó mediante comparaciones 2 a 2 para comprobar si las medianas eran diferentes, empleando el test U de Mann Whitney.

La población diana fue la de los alumnos de enfermería de la promoción 2007-2010 de la Escuela Universitaria de Enfermería de la Universidad San Pablo CEU ubicada en el Hospital Central de la Defensa «Gómez Ulla». Se comenzó con la población de alumnos de primer curso (2007-2008) a la que, de forma voluntaria y anónima, aproximadamente en el ecuador de sus prácticas clínicas, se le pasó el cuestionario.

Durante el primer curso los alumnos realizaron dos rotatorios en el área de hospitalización de nueve semanas de duración cada uno, teniendo como objetivo la práctica de cuidados básicos de enfermería. En el segundo curso realizaron cuatro rotatorios de ocho semanas cada uno: 3 Rotatorios en el área de hos- 

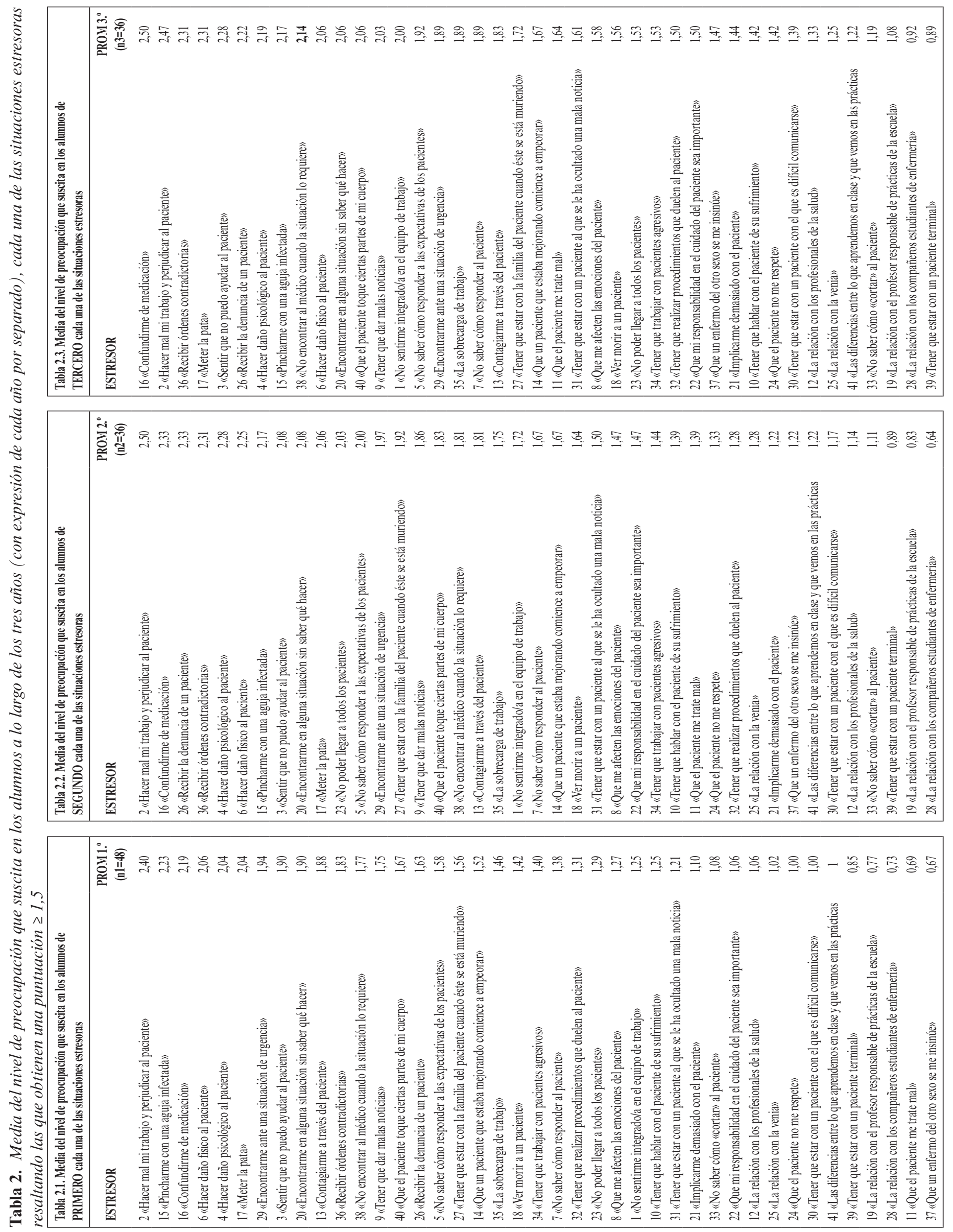
Identificación de estresores en las prácticas clínicas y evolución de los mismos en una promoción...

Tabla 3. Agrupación de estresores según los nueve factores o subescalas (KEZKAK) Comparativa entre primer, egundo y tercer año de carrera

\begin{tabular}{|c|c|c|c|c|}
\hline ESTRESOR & TOTAL (120) & $\begin{array}{c}\operatorname{PROM~1.} \\
(n 1=48)\end{array}$ & $\begin{array}{c}\text { PROM 2. } \\
\text { (n2=36) }\end{array}$ & $\begin{array}{c}\text { PROM 3. } \\
(n 3=36)\end{array}$ \\
\hline \multicolumn{5}{|l|}{ F1 - FALTA DE COMPETENCIA } \\
\hline 2 «Hacer mal mi trabajo y perjudicar al paciente» & 2,46 & 2,40 & 2,50 & 2,47 \\
\hline 16 «Confundirme de medicación» & 2,34 & 2,19 & 2,33 & 2,50 \\
\hline 15 «Pincharme con una aguja infectada» & 2,19 & 2,23 & 2,17 & 2,17 \\
\hline 4 «Hacer daño psicológico al paciente» & 2,17 & 2,04 & 2,28 & 2,19 \\
\hline 17 «Meter la pata» & 2,13 & 2,04 & 2,06 & 2,31 \\
\hline 6 «Hacer daño físico al paciente» & 2,12 & 2,06 & 2,25 & 2,06 \\
\hline 3 «Sentir que no puedo ayudar al paciente» & 2,09 & 1,90 & 2,08 & 2,28 \\
\hline 26 «Recibir la denuncia de un paciente» & 2,06 & 1,63 & 2,33 & 2,22 \\
\hline 13 «Contagiarme a través del paciente» & 1,84 & 1,88 & 1,81 & 1,83 \\
\hline 5 «No saber cómo responder a las expectativas de los pacientes» & 1,83 & 1,58 & 2,00 & 1,92 \\
\hline 1 «No sentirme integrado/a en el equipo de trabajo» & 1,66 & 1,25 & 1,72 & 2,00 \\
\hline \multicolumn{5}{|l|}{ F2-CONTACTO CON EL SUFRIMIENTO } \\
\hline 29 «Encontrarme ante una situación de urgencia» & 1,93 & 1,94 & 1,97 & 1,89 \\
\hline 9 «Tener que dar malas noticias» & 1,88 & 1,75 & 1,86 & 2,03 \\
\hline 27 «Tener que estar con la familia del paciente cuando éste se está muriendo» & 1,73 & 1,56 & 1,92 & 1,72 \\
\hline 14 «Que un paciente que estaba mejorando comience a empeorar» & 1,62 & 1,52 & 1,67 & 1,67 \\
\hline 18 «Ver morir a un paciente» & 1,54 & 1,42 & 1,64 & 1,56 \\
\hline 34 «Tener que trabajar con pacientes agresivos» & 1,46 & 1,40 & 1,44 & 1,53 \\
\hline 31 «Tener que estar con un paciente al que se le ha ocultado una mala noticia» & 1,44 & 1,21 & 1,50 & 1,61 \\
\hline 32 «Tener que realizar procedimientos que duelen al paciente» & 1,36 & 1,31 & 1,28 & 1,50 \\
\hline 10 «Tener que hablar con el paciente de su sufrimiento» & 1,35 & 1,25 & 1,39 & 1,42 \\
\hline 39 «Tener que estar con un paciente terminal» & 0,88 & 0,85 & 0,89 & 0,89 \\
\hline \multicolumn{5}{|l|}{ F3 - RELACIÓN CON TUTORES Y COMPAÑEROS } \\
\hline 20 «Encontrarme en alguna situación sin saber qué hacer» & 2,01 & 1,90 & 2,08 & 2,06 \\
\hline 1 «No sentirme integrado/a en el equipo de trabajo» & 1,66 & 1,25 & 1,72 & 2,00 \\
\hline 25 «La relación con la venia» & 1,18 & 1,02 & 1,28 & 1,25 \\
\hline 12 «La relación con los profesionales de la salud» & 1,18 & 1,06 & 1,14 & 1,33 \\
\hline 19 «La relación con el profesor responsable de prácticas de la escuela» & 0,90 & 0,77 & 0,83 & 1,08 \\
\hline 28 «a relación con los compañeros estudiantes de enfermería» & 0,76 & 0,73 & 0,64 & 0,92 \\
\hline \multicolumn{5}{|l|}{ F4 - IMPOTENCIA E INCERTIDUMBRE } \\
\hline 2 «Hacer mal mi trabajo y perjudicar al paciente» & 2,46 & 2,40 & 2,50 & 2,47 \\
\hline 36 «Recibir órdenes contradictorias» & 2,15 & 1,83 & 2,31 & 2,31 \\
\hline 17 «Meter la pata» & 2,13 & 2,04 & 2,06 & 2,31 \\
\hline 6 «Hacer daño físico al paciente» & 2,12 & 2,06 & 2,25 & 2,06 \\
\hline 3 «Sentir que no puedo ayudar al paciente» & 2,09 & 1,90 & 2,08 & 2,28 \\
\hline 20 «Encontrarme en alguna situación sin saber qué hacer» & 2,01 & 1,90 & 2,08 & 2,06 \\
\hline 38 «No encontrar al médico cuando la situación lo requiere» & 1,91 & 1,77 & 1,81 & 2,14 \\
\hline 14 «Que un paciente que estaba mejorando comience a empeorar» & 1,62 & 1,52 & 1,67 & 1,67 \\
\hline 23 «No poder llegar a todos los pacientes» & 1,62 & 1,29 & 2,03 & 1,53 \\
\hline 32 «Tener que realizar procedimientos que duelen al paciente» & 1,36 & 1,31 & 1,28 & 1,50 \\
\hline 41 «Las diferencias entre lo que aprendemos en clase y que vemos en las prácticas & 1,15 & 1,00 & 1,22 & 1,22 \\
\hline \multicolumn{5}{|l|}{ F5 - NO CONTROLAR LA RELACIÓN CON EL ENFERMO } \\
\hline 17 «Meter la pata» & 2,13 & 2,04 & 2,06 & 2,31 \\
\hline 20 «Encontrarme en alguna situación sin saber qué hacer» & 2,01 & 1,90 & 2,08 & 2,06 \\
\hline 29 «Encontrarme ante una situación de urgencia» & 1,93 & 1,94 & 1,97 & 1,89 \\
\hline 5 «No saber cómo responder a las expectativas de los pacientes» & 1,83 & 1,58 & 2,00 & 1,92 \\
\hline 7 «No saber cómo responder al paciente» & 1,64 & 1,38 & 1,67 & 1,89 \\
\hline 30 «Tener que estar con un paciente con el que es difícil comunicarse» & 1,19 & 1,00 & 1,17 & 1,39 \\
\hline 33 «No saber cómo «cortar» al paciente» & 1,13 & 1,08 & 1,11 & 1,19 \\
\hline 39 «Tener que estar con un paciente terminal» & 0,88 & 0,85 & 0,89 & 0,89 \\
\hline \multicolumn{5}{|l|}{ F6 - IMPLICACIÓN EMOCIONAL } \\
\hline 8 «Que me afecten las emociones del paciente» & 1,44 & 1,27 & 1,47 & 1,58 \\
\hline 31 «Tener que estar con un paciente al que se le ha ocultado una mala noticia» & 1,44 & 1,21 & 1,50 & 1,61 \\
\hline 22 «Que mi responsabilidad en el cuidado del paciente sea importante» & 1,34 & 1,06 & 1,47 & 1,50 \\
\hline 21 «mplicarme demasiado con el paciente» & 1,26 & 1,10 & 1,22 & 1,44 \\
\hline \multicolumn{5}{|l|}{ F7- DAÑARSE EN LA RELACIÓN CON EL ENFERMO } \\
\hline 15 «Pincharme con una aguja infectada» & 2,19 & 2,23 & 2,17 & 2,17 \\
\hline 26 «Recibir la denuncia de un paciente» & 2,06 & 1,63 & 2,33 & 2,22 \\
\hline 14 «Que un paciente que estaba mejorando comience a empeorar» & 1,62 & 1,52 & 1,67 & 1,67 \\
\hline 24 «Que el paciente no me respete» & 1,25 & 1,00 & 1,33 & 1,42 \\
\hline 11 «Que el paciente me trate mal» & 1,24 & 0,69 & 1,39 & 1,64 \\
\hline \multicolumn{5}{|l|}{ F8 - EL ENFERMO BUSCA UNA RELACIÓN ÍNTIMA } \\
\hline 40 «Que el paciente toque ciertas partes de mi cuerpo» & 1,85 & 1,67 & 1,83 & 2,06 \\
\hline 37 «Que un enfermo del otro sexo se me insinúe» & 1,12 & 0,67 & 1,22 & 1,47 \\
\hline \multicolumn{5}{|l|}{ F9 - SOBRECARGA } \\
\hline 36 «Recibir órdenes contradictorias» & 2,15 & 1,83 & 2,31 & 2,31 \\
\hline 35 «La sobrecarga de trabajo» & 1,70 & 1,46 & 1,75 & 1,89 \\
\hline 34 «Tener que trabajar con pacientes agresivos» & 1,46 & 1,40 & 1,44 & 1,53 \\
\hline 31 «Tener que estar con un paciente al que se le ha ocultado una mala noticia» & 1,44 & 1,21 & 1,50 & 1,61 \\
\hline 30 «Tener que estar con un paciente con el que es difícil comunicarse» & 1,19 & 1,00 & 1,17 & 1,39 \\
\hline
\end{tabular}


pitalización (Unidades de Hospitalización Médico-quirúrgica, Área Materno-infantil, Área Quirúrgica, Unidad de Hemodinámica, Unidad de Reconocimiento, Microbiología y Bioquímica clínica). 1 Rotatorio en Centros de Salud.

Por último, durante el tercer año de carrera realizaron cuatro rotatorios (dos de ocho semanas de duración y otros dos de siete semanas) en el área de hospitalización: Unidades de Hospitalización Médico-quirúrgica, Servicio de Oncología, Unidad de Geriatría, Unidad de Psiquiatría, Área Materno-infantil, Área Quirúrgica, UCI y Unidad de Coronarias, Urgencias, Unidad de Hemodiálisis.

Los datos fueron tratados estadísticamente por medio del paquete estadístico $\operatorname{SPSS}^{\circledR}$ v15.0.

\section{RESULTADOS}

Como no se avisó el día ni la actividad, entre la falta de asistencia y las pérdidas por deficiencias de cumplimentación, se consiguió una encuesta válida del $75 \%$ de la población diana (los 64 estudiantes de primer curso) $(n=48)$. En el segundo curso (2008-2009), utilizando la misma metodología de recogida de datos y en el mismo período del año académico, de los 60 alumnos matriculados se obtuvo un tamaño poblacional del $60 \%(n=36)$ del total. Y en el tercer y último año, de los 58 alumnos matriculados el porcentaje de participación fue del $62,06 \%(n=36)$ del total de alumnos del curso.

El rango de edad en primero se estableció entre 18 y 34 años (18-34) con una media de 20,31años $(3,82)$ y una mediana de 19. En segundo, el rango de edad (20-35) con una media de 22,36 (4) y una mediana de 20. El rango de edad en tercero [21-36] con una media de 23,39 años $(4,03)$ y una mediana de 21. La muestra en el primer curso estaba constituida por 45 mujeres y 3 hombres, en segundo por 35 mujeres y 2 hombres, y en el tercer curso por 34 mujeres y 2 hombres.

La relación entre el género y los resultados no se ha tenido en cuenta por estar compuesta la muestra mayoritariamente por mujeres, no siendo los resultados representativos respecto al género masculino.

Observando el promedio global en las respuestas de los tres cursos, encontramos 23 ítems cuyo valor promedio es superior a 1,5. Es decir, el promedio total emanado de las respuestas de los alumnos a lo largo de los tres años de carrera define a esos 23 estresores como los responsables de las tensiones sufridas durante sus prácticas clínicas (tabla 1), oscilando entre un valor promedio máximo de 2,46 y un promedio mínimo de 1,54. En el desglose por curso se observa mucho más detalladamente (tabla 2) que el número de estresores va aumentando curso a curso, con la particularidad de que los que aparecen en el último curso como menos significativos $(<1,5)$, no han sido significativos en ninguno de los cursos anteriores. Es decir, los estresores considerados por los alumnos de tercero como poco generadores de tensión durante sus prácticas clínicas, se mantuvieron en los mismos niveles poco significativos en los cursos previos.

De esos 23 estresores que se consideran como situaciones generadoras de tensión, 10 están puntuados entre 2 (bastante estrés) y 3 (mucho estrés). En general, vemos que las situaciones que más les preocupan y que coinciden en los tres cursos están en- cuadradas, por orden de mayor a menor, en las subescalas «falta de competencia», «impotencia e incertidumbre», «contacto con el sufrimiento» y «no controlar la relación con el enfermo» (tabla 5). Observando la evolución a lo largo de los tres años vemos que las puntuaciones en promedio indican un aumento desde primero hasta tercero que, como veremos en la discusión, es posible que tenga que ver con la distribución de los lugares en los que se realizan las prácticas a lo largo de los tres cursos.

Si comenzamos observando los 41 ítems del cuestionario KEZKAK (tabla 4) encontramos resultados estadísticamente significativos en los siguientes:

- Ítem $n .{ }^{o} 1$ «No sentirme integrado en el equipo de trabajo»: se observa variación a lo largo de la carrera en cuanto a la afectación por este ítem $(\mathrm{p}<0,01)$. El sentimiento de situación generadora de estrés manifestado en primero se ve aumentado en el 2. ${ }^{\circ}$ año incrementándose el valor de este ítem en casi 0,5 puntos respecto al obtenido en $1 .^{\circ}(\mathrm{p}<0,05)$, y un incremento de 0,75 desde $1^{\circ}$ a $3^{\circ}(\mathrm{p}<0,001)$.

- Ítem $n .{ }^{\circ} 3$ «Sentir que no puedo ayudar al paciente»: se observa un incremento de la media de casi 0,4 puntos como situación generadora de tensión en $3 .^{\circ}$ respecto al 1 . $^{\text {er }}$ año $(p<0,05)$. Sin embargo no existe diferencia estadísticamente significativa en la opinión a este respecto entre $1 .^{\circ}$ y $2 .^{\circ}$, ni entre $2 .^{\circ}$ y $3 .^{\circ}$

- Ítem $n .^{\circ} 7$ «No saber cómo responder al paciente»: se observa variación a lo largo de la carrera en cuanto a la afectación por este ítem $(p<0,05)$. Al igual que en el anterior se observa significación estadística $(p<0,05)$ en el sentido de un incremento de la afectación de los alumnos por este estresor en el 3. ${ }^{\text {er }}$ año respecto al $1 .^{\circ}$ con un aumento de la media de 0,51 .

- Ítem $n .^{\circ} 11$ «Que el paciente me trate mal»: se observa variación a lo largo de la carrera en cuanto a la afectación por este ítem $(\mathrm{p}<0,001)$. El sentimiento de situación generadora de estrés manifestado en primero se ve aumentado en el $2 .^{\circ}$ año incrementándose el valor de este ítem en 0,7 puntos respecto al obtenido en $1 .^{\circ}(\mathrm{p}<0,01)$, y un incremento de 0,95 desde $1 .^{\circ}$ a $3 .^{\circ}$ $(\mathrm{p}<0,001)$.

- Ítem $n .^{\circ} 16$ «Confundirme de medicación»: se observa un incremento de la media de 0,31 puntos como situación generadora de tensión en $3 .^{\circ}$ respecto al $1^{\text {er }}$ año $(\mathrm{p}<0,05)$.

- Ítem $n .^{\circ} 22$ «Que mi responsabilidad en el cuidado del paciente sea importante»: el estrés que genera este ítem en primero se ve aumentado en el $2 .^{\circ}$ año incrementándose el valor de este ítem en 0,3 puntos $(\mathrm{p}<0,05)$, y un incremento de 0,4 desde $1 .^{\circ}$ a 3. ${ }^{\circ}(\mathrm{p}<0,05)$.

- Ítem $n .^{\circ} 24$ "Que el paciente no me respete»: se observa un incremento de la media de 0,4 puntos como situación generadora de tensión en $3 .^{\circ}$ respecto al $1 .^{\text {er }}$ año $(\mathrm{p}<0,05)$.

- Ítem $n .^{\circ} 26$ «Recibir la denuncia de un paciente»: se observa variación a lo largo de la carrera en cuanto a la afectación por este ítem $(\mathrm{p}<0,01)$. La percepción por parte de los alumnos de situación generadora de estrés en primero se ve aumentado en el $2 .^{\circ}$ año incrementándose el valor de este ítem en 0,7 puntos respecto al obtenido en $1 .^{\circ}(\mathrm{p}<0,01)$, y un incremento de 0,7 desde $1 .^{\circ}$ a $3 .^{\circ}(\mathrm{p}<0,05)$.

- Ítem $n .^{\circ} 30$ «Tener que estar con un paciente con el que es difícil comunicarse»: se observa un incremento de la media de 0,4 puntos como situación generadora de tensión en $3 .^{\circ}$ respecto al $1 .{ }^{\text {er }}$ año $(\mathrm{p}<0,05)$. 


\section{Identificación de estresores en las prácticas clínicas y evolución de los mismos en una promoción...}

Tabla 4. Media(desviación típica)/mediana (rango intercuartilico) de cada item de la encuesta Kezkak, totales y en cada curso. Estudio comparativo de cada item entre los cursos dos a dos -tets U Mann-Whitney y entre los 3 cursos -test de Kruskal Wallis

\begin{tabular}{|c|c|c|c|c|c|c|c|c|}
\hline Cuestionario KEZKAK & Total & Primer curso & Segundo curso & Tercer curso & $\mathrm{U} 1 .{ }^{\circ} / 2 .^{\circ}$ & $\mathrm{U} 1 .^{\circ} / 3 .^{\circ}$ & $\mathrm{U} 2 .^{\circ} / 3 .^{\circ}$ & $\begin{array}{c}\mathrm{KW} \\
1 .{ }^{\prime} / 2 .^{\circ} /{ }^{\circ}{ }^{\circ}\end{array}$ \\
\hline 1 «No sentirme integrado/a en el equipo de trabajo» & $1,62(0,92) / 2(1)$ & $1,25(0,9) / 1(2)$ & $1,72(0,9) / 2(2)$ & $2(0,76) / 2(0)$ & $*$ & $* * *$ & & $* *$ \\
\hline 2 «Hacer mal mi trabajo y perjudicar al paciente» & $2,45(0,74) / 3(1)$ & $2,4(0,8) / 3(1)$ & $2,5(0,74) / 3(1)$ & $2,47(0,74) / 3(1)$ & & & & \\
\hline 3 «Sentir que no puedo ayudar al paciente» & $2,07(0,79) / 2(1)$ & $1,9(0,8) / 2(1)$ & $2,1(0,7) / 2(1)$ & $2,28(0,82) / 2(1)$ & & $*$ & & \\
\hline 4 «Hacer daño psicológico al paciente» & $2,16(085) / 2(1)$ & $2,04(0,9) / 2(1)$ & $2,3(0,8) / 2(1)$ & $2,19(0,82) / 2(1)$ & & & & \\
\hline 5 «No saber cómo responder a las expectativas de los pacientes» & $1,81(0,82) / 2(1)$ & $1,58(0,85) / 2(1)$ & $2(0,76) / 2(2)$ & $1,92(0,81) / 2(2)$ & & & & \\
\hline 6 «Hacer daño físico al paciente» & $2,12(0,85) / 2(1)$ & $2,06(0,91) / 2(2)$ & $2,25(0,7) / 2(1)$ & $2,06(0,89) / 2(2)$ & & & & \\
\hline 7 «No saber cómo responder al paciente» & $1,62(0,88) / 2(1)$ & $1,38(0,84) / 1(1)$ & $1,7(0,8) / 2(1)$ & $1,89(0,92) / 2(2)$ & & $*$ & & * \\
\hline 8 «Que me afecten las emociones del paciente» & $1,43(0,93) / 1$ & $(1) 1,27(0,96) / 1(2)$ & $1,47(0,9) / 1(1)$ & $1,58(0,9) / 1(1)$ & & & & \\
\hline 9 «Tener que dar malas noticias» & $1,87(0,94) / 2(2)$ & $1,75(0,89) / 2(1)$ & $1,86(1) / 2(2)$ & $2,03(094) / 2(2)$ & & & & \\
\hline 10 «Tener que hablar con el paciente de su sufrimiento» & $1,34(0,90) / 1(1)$ & $1,25(0,86) / 1(1)$ & $1,39(0,9) / 1(1)$ & $1,42(0,94) / 1(1)$ & & & & \\
\hline 11 «Que el paciente me trate mal» & $1,18(1,02) / 1(2)$ & $0,69(0,8) / 1(1)$ & $1,39(1,1) / 1(2)$ & $1,64(0,96) / 2(1)$ & $* *$ & $* * *$ & & $* * *$ \\
\hline 12 «La relación con los profesionales de la salud» & $1,17(0,92) / 1(2)$ & $1,06(0,98) / 1(2)$ & $1,14(0,8) / 1(1)$ & $1,33(0,96) / 1(1)$ & & & & \\
\hline 13 «Contagiarme a través del paciente» & $1,84(0,98) / 2(2)$ & $1,88(1,1) / 2(2)$ & $1,81(0,89) / 2(2)$ & $1,83(0,97) / 2(2)$ & & & & \\
\hline 14 «Que un paciente que estaba mejorando comience a empeorar» & $1,61(0,81) / 2(1)$ & $1,52(0,9) / 1(1)$ & $1,67(0,76) / 2(1)$ & $1,67(0,79) / 2(1)$ & & & & \\
\hline 15 «Pincharme con una aguja infectada» & $2,19(0,91) / 2(2)$ & $2,23(0,9) / 2,5(1)$ & $2,17(0,98) / 2,5(2)$ & $2,17(0,88) / 2(2)$ & & & & \\
\hline 16 «Confundirme de medicación» & $2,33(0,76) / 2(1)$ & $2,19(0,7) / 2(1)$ & $2,33(0,87) / 3(1)$ & $2,5(0,7) / 3(1)$ & & $*$ & & \\
\hline 17 «Meter la pata» & $2,13(0,86) / 2(1)$ & $2,04(0,8) / 2(1)$ & $2,1(1) / 2(2) 2,31$ & $(0,79) / 2,5(1)$ & & & & \\
\hline 18 «Ver morir a un paciente» & $1,53(0,97) / 2(1)$ & $1,42(1,1) / 1(2)$ & $1,64(0,9) / 2(1)$ & $1,56(0,84) / 2(1)$ & & & & \\
\hline 19 «La relación con el profesor responsable de prácticas de la escuela» & $0,88(0,89) / 1(1)$ & $0,77(0,86) / 1(1)$ & $0,83(0,9) / 1(1)$ & $1,1(0,91) / 1(2)$ & & & & \\
\hline 20 «Encontrarme en alguna situación sin saber qué hacer» & $2,0(0,81) / 2(2)$ & $1,9(0,86) / 2(2)$ & $2,1(0,84) / 2(2)$ & $2,06(0,72) / 2(1)$ & & & & \\
\hline 21 «mplicarme demasiado con el paciente» & $1,24(0,92) / 1(1)$ & $1,1(0,93) / 1(2)$ & $1,22(0,9) / 1(1)$ & $1,44(0,91) / 1(1)$ & & & & \\
\hline 22 «Que mi responsabilidad en el cuidado del paciente sea importante» & $1,32(0,93) / 1(1)$ & $1,06(0,86) / 1(2)$ & $1,47(1) / 2(1)$ & $1,5(0,77) / 1(1)$ & $*$ & * & & \\
\hline 23 «No poder llegar a todos los pacientes» & $1,43(0,94) / 1(1)$ & $1,29(1) / 1(2)$ & $1,5(0,91) / 1(1)$ & $1,53(0,91) / 2(1)$ & & & & \\
\hline 24 «Que el paciente no me respete» & $1,23(0,93) / 1(2)$ & $1,02(0,91) / 1(2)$ & $1,33(1) / 1(2)$ & $1,42(0,84) / 1(1)$ & & $*$ & & \\
\hline 25 «La relación con la venia» & $1,17(0,94) / 1(2)$ & $1,02(0,86) / 1(2)$ & $1,28(0,97) / 1(1)$ & $1,25(1) / 1(1)$ & & & & \\
\hline 26 «Recibir la denuncia de un paciente» & $2,02(1,04) / 2(2)$ & $1,63(1,1) / 2(2)$ & $2,33(0,87) / 3(1)$ & $2,22(0,93) / 2,5(1)$ & ** & $*$ & & $* *$ \\
\hline 27 «Tener que estar con la familia del paciente cuando éste se está muriendo» & $1,72(0,96) / 2(1)$ & $1,56(0,94) / 2(1)$ & $1,92(1) / 2(2)$ & $1,72(0,95) / 2(1)$ & & & & \\
\hline 28 «La relación con los compañeros estudiantes de enfermería» & $0,76(0,81) / 1(1)$ & $0,73(0,84) / 0,5(1)$ & $0,64(0,72) / 0,5(1)$ & $0,92(0,84) / 1(2)$ & & & & \\
\hline 29 «Encontrame ante una situación de urgencia» & $1,93(0,94) / 2(2)$ & $1,94(0,9) / 2(2)$ & $1,97(0,97) / 2(2)$ & $1,89(0,95) / 2(2)$ & & & & \\
\hline 30 «Tener que estar con un paciente con el que es difícil comunicarse» & $1,17(0,83) / 1(1)$ & $1(0,9) / 1(2)$ & $1,17(0,7) / 1(1)$ & $1,39(0,8) / 1(1)$ & & $*$ & & \\
\hline 31 «Tener que estar con un paciente al que se le ha ocultado una mala noticia» & $1,42(0,86) / 1(1)$ & $1,21(0,9) / 1(2)$ & $1,5(0,8) / 2(1)$ & $1,61(0,8) / 2(1)$ & & & & \\
\hline 32 «Tener que realizar procedimientos que duelen al paciente» & $1,36(0,86) / 1(1)$ & $1,3(0,9) / 1(1)$ & $1,28(0,81) / 1(1)$ & $1,50(0,81) / 1(1)$ & & & & \\
\hline 33 «No saber cómo «cortar» al paciente» & $1,13(0,92) / 1(2)$ & $1,08(0,96) / 1(2)$ & $1,1(0,92) / 1(2)$ & $1,19(0,89) / 1(1)$ & & & & \\
\hline 34 «Tener que trabajar con pacientes agresivos» & $1,45(0,92) / 1(1)$ & $1,4(1,03) / 1(1)$ & $1,44(0,91) / 2(1)$ & $1,53(0,77) / 1(1)$ & & & & \\
\hline 35 «La sobrecarga de trabajo» & $1,68(0,9) / 2(1)$ & $1,46(0,94) / 1(1)$ & $1,75(0,84) / 2(1)$ & $1,89(0,85) / 2(2)$ & & $*$ & & \\
\hline 36 «Recibir órdenes contradictorias» & $2,12(0,88) / 2(1)$ & $1,83(0,98) / 2(2)$ & $2,3(0,7) / 2(1)$ & $2,31(0,82) / 2(1)$ & $*$ & $*$ & & * \\
\hline 37 «Que un enfermo del otro sexo se me insinúe» & $1,08(1,05) / 1(2)$ & $0,67(0,93) / 0(1)$ & $1,22(0,9) / 1(2)$ & $1,47(1,1) / 2(2)$ & $* *$ & $* *$ & & $* *$ \\
\hline 38 «No encontrar al médico cuando la situación lo requiere» & $1,89(09) / 2(2)$ & $1,77(0,91) / 2(1)$ & $1,81(0,95) / 2(2)$ & $2,14(0,8) / 2(1)$ & & & & \\
\hline 39 «Tener que estar con un paciente terminal» & $0,88(0,91) / 1(1)$ & $0,85(0,92) / 1(1)$ & $0,89(0,98) / 1(1)$ & $0,89(0,85) / 1(1)$ & & & & \\
\hline 40 «Que el paciente toque ciertas partes de mi cuerpo» & $1,83(1,12) / 2(2)$ & $1,67(1,2) / 2(2)$ & $1,83(1,16) / 2(2)$ & $2,06(0,96) / 2(2)$ & & & & \\
\hline 41 «Las diferencias entre lo que aprendemos en clase y que vemos en las prácticas» & $1,13(0,94) / 1(2)$ & $1(0,9) 11(2)$ & $1,22(1,1) / 1(2)$ & $1,2(0,93) / 1(1)$ & & & & \\
\hline
\end{tabular}

${ }^{*} \mathrm{p}<0,05 ;{ }^{* *} \mathrm{p}<0,01 ;{ }^{* * *} \mathrm{p}<0,001$.

- Ítem $n .^{\circ} 35$ «La sobrecarga de trabajo»: hay un incremento de la media de 0,4 puntos como situación estresora en $3 .^{\circ}$ respecto al $1^{\text {er }}$ año $(\mathrm{p}<0,05)$.

- Ítem $n .^{\circ} 36$ «Recibir órdenes contradictorias»: se observa variación a lo largo de la carrera en cuanto a la afectación por este ítem $(\mathrm{p}<0,05)$. El sentimiento que los alumnos manifiestan en primero se ve aumentado en el $2 .^{\circ}$ año en casi 0,5 puntos respecto al obtenido en $1 .^{\circ}(\mathrm{p}<0,05)$, y un incremento igual desde 1. ${ }^{\mathrm{o}}$ a $3 .^{\circ}(\mathrm{p}<0,05)$.

- Ítem $n .^{\circ} 37$ "Que el enfermo se me insinúe»: se observa variación a lo largo de la carrera en cuanto a la afectación por este ítem $(\mathrm{p}<0,01)$. El sentimiento de situación generadora de estrés manifestado en primero se ve aumentado en el $2 .^{\circ}$ año incrementándose el valor de este ítem en casi 0,6 puntos respecto al obtenido en $1 .^{\circ}(\mathrm{p}<0,01)$, y un incremento de 0,8 desde $1 .^{\circ}$ a $3 .^{\circ}(\mathrm{p}<0,001)$.

Por último, haciendo el estudio comparativo entre cada una de las nueve subescalas (F1 a F9) (tabla 5), en general no se encontraron diferencias estadísticamente significativas en las respuestas a lo largo de los tres cursos de los mismos alumnos durante la realización de la diplomatura $(p>0,05)$. La única variación de manifestó en la subescala «el enfermo busca una relación íntima» (F8). Mientras los alumnos emitieron una puntuación de mediana $=2$ en el primer curso, dicha calificación de duplicó al finalizar sus estudios $(\mathrm{p}<0,05)$.

La incidencia de mayor a menor preocupación de los alumnos en sus prácticas clínicas en relación con los 9 factores o subescalas como fuentes generadoras de estrés fue (tabla 5): F1-falta de competencia $(24,56)$, F4-impotencia e incertidumbre $(20,29)$, F2-contacto con el sufrimiento (15,09), F5-no controlar la relación con el enfermo $(12,65)$, F7-dañarse en la relación con el enfermo (8,23), F9-sobrecarga (7,83), F3-relación con tutores y compañeros (7,59), F8-implicación emocional $(5,40)$ y F9-el enfermo busca una relación íntima $(2,91)$. En los estudios de referencia, este estudio coincide respecto a los dos factores de mayor incidencia. 
Tabla 5. Media (desviación típica)/mediana (rango intercuartílico) de cada grupo de ítems de la encuesta Kezkaz, totales y en cada curso. Estudio comparativo de cada grupo de items entre los cursos dos a dos-contrastes múltiples post hoc de Bonferroni-y entre los 3 cursos-ANOVA de una vía-

\begin{tabular}{|c|c|c|c|c|c|c|c|c|}
\hline \multirow{2}{*}{ Cuestionario KEZKAK por grupos } & \multirow{2}{*}{ Total } & \multirow{2}{*}{ Primer curso $\left(1 .^{\circ}\right)$} & \multirow{2}{*}{ Segudo curso $\left(2 .^{\circ}\right)$} & \multirow{2}{*}{ Tercer curso $\left(3 .^{\circ}\right)$} & \multicolumn{3}{|c|}{ Contraster múltiples Bonferroni } & \multirow{2}{*}{$\begin{array}{l}\text { ANOVA } \\
1 .^{\circ} / 2 .^{\circ} / 3 .^{\circ}\end{array}$} \\
\hline & & & & & $1 .{ }^{\circ} / 2 .^{\circ}$ & $1 .{ }^{\circ} / 3 .^{\circ}$ & $2 .^{\circ} / 3 .^{\circ}$ & \\
\hline Falta de competencia & $24,56(9,7) / 26(16)$ & $23,1(10,1) / 24,5(17)$ & $25,3(9,5) / 27,5(16)$ & $25,8(9,4) 127(15)$ & & & & \\
\hline Contacto con el sufrimiento & $15,09(8,5) 116(13)$ & $14,2(8,9) 113(13)$ & $15,56(8,5) 116(13)$ & $15,81(8,1) 116(12)$ & & & & \\
\hline Relación con tutores y campaneros & $7,59(4,9) 18(8)$ & $6,73(5,0) 16,5(10)$ & $7,7(4,8) 17,5(7)$ & $8,64(4,9) 18(7)$ & & & & \\
\hline Impotencia e incertidumbre & $20,29(8,7) 120(14)$ & 19,02(9)119(16) & $20,75(8,6) / 20(14)$ & $21,53(8,4) / 21,5(12)$ & & & & \\
\hline No controlar la relación con el enfermo & $12,65(6,5) 113(11)$ & $11,8(6,6) 112(12)$ & $12,94(6,5) 113(12)$ & $13,53(6,3) 113,5(11)$ & & & & \\
\hline Implicación emocional & $5,40(3,5) 14(4)$ & $4,65(3,6) 14(8)$ & $5,67(3,4) 16(4)$ & $6,14(3,4) 15(4)$ & & & & \\
\hline Dañarse en la relación con el enfermo & $8,23(4,4) 19(8)$ & $7,08(4,3) 7,5(7)$ & $8,89(4,4) 19,5(8)$ & $9,11(4,2) 19,5(6)$ & & & & \\
\hline El enfermo busca una relación intima & $2,91(2,1) 13(4)$ & $2,33(2) / 2(3)$ & $3,06(2,1) 13(4)$ & $3,53(198) 14(4)$ & & $*$ & & $*$ \\
\hline Sobrecarga & $7,83(4,1) 18(5)$ & $6,9(4,6) 16(8)$ & $8,17(3,7) 18(5)$ & $8,72(3,8) 18(6)$ & & & & \\
\hline
\end{tabular}

$* \mathrm{p}<0,05$.

\section{DISCUSIÓN}

Al igual que en los estudios de referencia ${ }^{14,15}$ la participación ha sido significativa. En el primer curso hay una participación alta, produciéndose una pérdida importante de encuestas a lo largo de la carrera, siendo esto debido a que los alumnos, según avanzaban los cursos y en relación a temarios más exigentes, mayor número de asignaturas, junto con las prácticas clínicas fuera del hospital, se organizaron en grupos de trabajo de manera que se repartían los días de asistencia a clase entre los miembros del grupo.

Sí se plantearon diferencias referentes a los resultados obtenidos en las puntuaciones de los 9 factores o subescalas respecto al curso académico: mientras que en los estudios de referencia la puntuación en $2 .^{\circ}$ curso fue superior a la de los otros dos, en el presente trabajo, globalmente las puntuaciones tendieron a aumentar desde primero a tercero (tabla 5). No existen datos en los estudios de referencia de la distribución de los servicios extrahospitalarios y hospitalarios por los que pasaron los alumnos en cada uno de los cursos. En este sentido, en el presente estudio sí podemos observar que los rotatorios por los servicios que podían generar más tensiones en los alumnos (oncología, urgencias, coronarias, UCI, psiquiatría) se realizaron en el último curso.

Analizando los factores o subescalas más significativos, de mayor a menor puntuación según la opinión de los alumnos, y los ítems de los que están compuestos vemos que:

- La falta de competencia (F1) es el factor más ponderado y aglutina ítems relacionados con el temor a dañar, o no poder ayudar al paciente o a resultar dañado. Al alumno le parece estresante la posibilidad de no realizar bien su trabajo y las consecuencias que ello puede acarrear tanto para el paciente como para él mismo. Es un factor que está presente en los alumnos y que puede dejar de ser tan estresante a medida que se va adquiriendo destreza y experiencia. En nuestro caso, en la puntuación global del factor esto no ocurre (tabla 5), pero 5 de los 11 ítems que lo forman si siguen esta tendencia de disminución respecto al tercer año (tabla 3). Como medidas de apoyo, consideramos necesario:

- Centrar al alumno en su rol, de manera que la exigencia en su formación práctica se ajuste año a año a esa condición de alumno. Ya sea por medio de la información de cuáles son las fuentes de estrés a las que se van a ser sometidos, como a las herramientas para saber afrontarlas, como a convencerlos de que eviten grados de autoexigencia superiores a los que están preparados.

- Minimizar la incidencia de los efectos de esas fuentes de estrés, informando a los alumnos de la normalidad de su aparición, de la realidad de que con la experiencia y el aprendizaje algunas de ellas desaparecerán, y de que las que se mantienen a lo largo de la vida profesional se pueden minimizar por medio del entrenamiento de estrategias específicas (sesiones clínicas, supervisiones de grupo, grupos de autoapoyo, trabajo en equipo, etc.).

- El siguiente factor más valorado en cuanto a las tensiones que genera en los alumnos es la impotencia e incertidumbre (F4). En el deseo de ayudar a los demás nos gustaría ser omnipotentes, pero la realidad frustra este deseo muy frecuentemente. Observamos que algunos ítems generan tensión aumentada en $2 .^{\circ}$ curso respecto a $1 .^{\circ}$, pero este sentimiento disminuye en $3 .^{\circ}$ (hacer mal mi trabajo y perjudicar al paciente, hacer daño físico al paciente, encontrarme una situación sin saber qué hacer, no poder llegar a todos los pacientes) lo cual significa que el paso del tiempo y la experiencia adquirida va haciendo que se vayan dominando situaciones que resultaban más problemáticas en el curso anterior. Es destacable en este factor la poca relevancia y la falta de significación estadística que tiene en nuestro estudio, a diferencia de los de referencia, el ítem relacionado con la preocupación de que haya diferencias entre lo aprendido en la escuela y lo que se realiza en las prácticas clínicas. Se trata de un buen referente de la calidad y conjunción de la enseñanza teórica y su traducción en la práctica.

- El contacto con el sufrimiento (F2) sigue en incidencia como factor generador de tensiones en las prácticas clínicas de los alumnos de nuestra escuela. Dentro de esta subescala el aspecto de la muerte y el proceso de morir quizá sea lo más relevante en cuanto a la generación de estrés. En referencia a la actitud ante la muerte es significativo en nuestro estudio la preocupación de los alumnos en cuanto al momento inminente de la muerte y el enfrentarse a los sentimientos de las personas allegadas (ver morir a un paciente $[1,54]$ y tener que estar con la familia del paciente cuando este se está muriendo [1,73]). Sin embargo cuando no existe la inminencia, les preocupa menos (tener que estar con un paciente terminal $[0,88]$ ). En todo caso, todos los ítems a los que me he referido, manifiestan su puntuación más alta en segundo y más baja en primero y tercero. Esto puede ser debido a que en primero aún no han tenido contacto con esta realidad y en tercero ya pueden haberla experimentado 


\section{Identificación de estresores en las prácticas clínicas y evolución de los mismos en una promoción...}

y, además, haber sido aconsejados. Es decir, esta disminución en el último año de carrera puede deberse, en parte, a la experiencia y a la formación.

- No controlar la relación con el enfermo (F5) es un factor que no sólo está presente en los alumnos de enfermería de nuestra escuela y en los de los estudios de referencia, sino que también es una fuente de estrés importante en profesionales de enfermería ${ }^{15,16}$.

- «Meter la pata» y «encontrarme en alguna situación sin saber que hacer» son las dos situaciones que, en valoración absoluta, preocupan bastante a nuestros alumnos. Casi todos los ítems que constituyen esta fuente de estrés tienen una valoración creciente a lo largo de los tres años de carrera. En este sentido, el desarrollo de habilidades sociales es de capital importancia para realizar el trabajo de enfermería. Es básico que el alumno sepa que la relación con el paciente es fundamental en su profesión y que debe conocer las habilidades sociales para ir desarrollándolas paulatinamente. Dependiendo del nivel de desarrollo de sus habilidades, habrá que hacer hincapié al alumno, que la actividad le indicará el tipo de relación a establecer y la «distancia de seguridad» a mantener para evitar la implicación emocional con el paciente.

- Dañarse en la relación con el enfermo (F7) presenta una carga importante de estrés en relación a «pincharse con una aguja infectada» $\mathrm{y}$ 《recibir la denuncia de un paciente» $(>2)$. Se trata de estresores que alcanzan su punto álgido en segundo, disminuyendo en tercero, quizá por la experiencia y adquisición de estrategias. Importante el aumento en tercero del nivel de estrés que produce el ítem «que el paciente me trate mal»; relacionado con la rotación por el servicio de psiquiatría en dicho curso.

Existen factores que no desaparecen con el tiempo ni con la experiencia: implicación emocional, contacto con el sufrimiento y la impotencia e incertidumbre ${ }^{16}$. Respecto a ellos, conviene hacer ver a los alumnos que se trata de fuentes de estrés que acompañan al trabajo de enfermería y la conveniencia de ir familiarizándose para vivir con ellas y poder trabajarlas. La escuela debe fomentar la creación de espacios de reflexión en torno a las prácticas clínicas. En estos espacios, el alumno puede aprender a afrontar estas situaciones mediante el desahogo con sus compañeros y su apoyo (universalización), comprobando que todos están sufriendo experiencias parecidas, pudiendo contrastar opiniones e intentando comprender lo que sucede.

Respecto a los factores «relación con tutores y compañeros» y «la sobrecarga», tan sólo remarcar la poca incidencia como fuente de estrés y en los dos ítems en los que se manifiesta una mayor puntuación su incremento en segundo curso y su mantenimiento o decrecimiento en tercero. En cuanto a la relación con tutores y compañeros, se trata de una fuente de estrés importante en profesionales de enfermería que tiene que ver con las dificultades en las relaciones con los compañeros y la jerarquía ${ }^{17}$. Enseñar a los alumnos a trabajar en equipo puede paliar, en cierta medida, las situaciones generadas en un futuro por esta fuente de estrés.

Por último, respecto al factor «el enfermo busca una relación íntima», en este estudio es la única fuente de estrés cuya variación a lo largo de la carrera tiene significación estadística. La explicación se encuentra en la distribución de los rotatorios de prácticas a lo largo de los tres cursos. En tercero, se produce el rotatorio por el servicio de psiquiatría, lugar en donde existe una gran incidencia de búsqueda de contacto físico por parte de los pacientes ingresados.

\section{CONCLUSIONES}

A la luz de los resultados y de la discusión de los mismos, se puede concluir afirmando que las principales fuentes de estrés que afectan a los alumnos de nuestra escuela coinciden en cuanto a intensidad y prioridad con los estudios precedentes usados como referencia. Sin embargo, en general, van aumentando en intensidad al transcurrir cada curso como consecuencia de la distribución de los rotatorios de prácticas a lo largo de los tres años de carrera, ya que los rotatorios por los servicios que podían generar más tensiones en los alumnos (oncología, urgencias, coronarias, UCI, psiquiatría) se realizan en el último año.

\section{AGRADECIMIENTOS}

A Carlos Gutiérrez Ortega, por su amistad y colaboración siempre desinteresada.

\section{BIBLIOGRAFÍA}

1. Martín IM. Estrés académico en estudiantes universitarios. Apuntes de Psicología. 2007; 25(1): 87-99.

2. Labrador FJ, Crespo M. Estrés. Trastornos psicofisiológicos. Madrid. Eudema; 1993.

3. Hernández JM, Pozo C. y Polo A. La ansiedad ante los exámenes. Un programa para su tratamiento de forma eficaz. Valencia. Promolibro; 1994.

4. Muñoz FJ. El estrés académico. Problemas y soluciones desde una perspectiva psicosocial. Huelva. Publicaciones Universidad de Huelva; 2003.

5. Pellicer O, Salvador A, Benet I. Efectos de un estresor académico sobre la respuesta psicológica e inmune en jóvenes. 2002; 14: 317-322.

6. Zupiria, X. Fenómenos psicológicos emergentes en el comienzo de las prácticas hospitalarias en estudiantes de primero de enfermería. Boletín de la APAG. 1999; 13:45-52.

7. Zupiria X. Relación enfermo-profesional de la salud. Bilbao. Universidad del País Vasco. 2000; 166-178.

8. Tizón JL. Componentes psicológicos de la práctica médica. Barcelona. Doyma. 1988.

9. Zupiria X., Uranga M.J., Alberdi M.J. y cols. Fuentes de estrés en la práctica clínica de los estudiantes de enfermería. Evolución a lo largo de la diplomatura. Enferm Clin. 2006; 16:231-237.

10. Alves Apóstolo J, Alves Rodrigues M, Pineda J. Evaluación de los estados emocionales de estudiantes de enfermería. Index de Enfermería (edición digital). 2007 [consulta el 05 febrero de 2012]; 16(56). Disponible en: http://scielo.isciii.es/scielo.php?pid=S113212962007000100006\&script $=$ s ci_arttext\&tlng=pt

11. López-Medina IM. Et al. Percepción del estrés en estudiantes de enfermería en las prácticas clínicas. Enfermería Clínica. 2005; 15(6): 307-313.

12. Zupiria X, Uranga MJ, Alberdi MJ. y cols. Fuentes de estrés en la práctica clínica de los estudiantes de enfermería. Evolución a lo largo de la diplomatura. Op.cit.

13. Antolin R, Puialto MJ, Moure ML, Quinteiro T. Situaciones en las prácticas clínicas que provocan estrés en los estudiantes de enfermería. Enfermería Global. 2007; 10:1-12.

14. Zupiria X., Uranga M.J., Alberdi M.J. y cols. KEZKAK: cuestionario bilingüe de estresores de los estudiantes de enfermería en las prácticas clínicas. Gac Sanit. 2003; 17(1): 37-51.

15. Tomás J, Fernández L. Estrés laboral en los profesionales de enfermería hospitalarios. Metas de Enfermería. 2002; 5:6-12.

16. McGrath A, Reid N, Boore J. Occupational stress in nursing. Int J Nurs Stud. 2003; 40:555-565.

17. Gray-Toft P, Anderson, JG. The Nursing Stress Scale: development of an instrument. J Behav Asses. 1981; 3: 1-23. 